\title{
INTELLIGENT ADAPTIVE SCHEDULERS FOR RAILWAYS
}

\author{
G. RZEVSKI ${ }^{1} \&$ P. SKOBELEV ${ }^{2}$ \\ ${ }^{1}$ The Open University, UK and Multi-Agent Technology Ltd, UK. \\ ${ }^{2}$ Samara University and Smart Solutions Ltd, Samara, Russia.
}

\begin{abstract}
Railway operation can be perceived as a complex system consisting of thousands of constituent elements, including demands for transportation and a variety of transportation resources, such as tracks, track blocks, stations, sidings, trains, crews, etc. Overall behaviour of a railway operation is characterized by unpredictable disruptive events such as changes in availability of resources due to failures, weather conditions or human errors. In addition transportation demands tend to change over time whilst changing transportation resources, such as tracks, is not always possible or practical. The key task of railway management is the allocation of transportation resources to transportation demands with a goal of achieving a complete match, ensuring a smooth operation. The difficulty of this task primarily depends on the variability of demand and reliability of resources, in particular, tracks, trains and human resources. The paper describes how to design complex adaptive railway schedulers, which allocate resources to demands in real time and ensure rapid rescheduling in reaction to unpredictable disruptive events.
\end{abstract}

Keywords: adaptability, complexity, railway schedules, real-time schedulers, self-organization.

\section{INTRODUCTION}

This paper describes how concepts, principles and methods of complexity science have been used to revolutionize timetabling and scheduling of railways.

\subsection{Complexity science basics}

Complexity is a property of open systems consisting of a large number of diverse, partially autonomous components, called agents, engaged in interaction by exchanging information, without having central control. Global behaviour of complex systems emerges from the interaction of agents and is unpredictable but not random; it follows discernible patterns.

There is a well-developed theory of complex systems [1-4], which provides rich variety of methods and tools for modelling and managing complexity [5], and documented experience in applying these methods and tools to variety of business and engineering problems, including real-time space logistics [6], real-time aircraft life cycle management [7], real-time supply chain management [8], real-time taxi scheduling [9], real-time car rental scheduling [10] and many others.

\subsection{Railway operation as a complex system}

Railway operation can be perceived as a complex system. It consists of thousands of interacting, diverse constituent elements, including demands for transportation and transportation resources such as tracks, track blocks, stations, station crews, trains, train crews, resource

This paper is part of the proceedings of the 15th International Conference on Railway

Engineering Design and Operation (COMPRAIL)

www.witconferences.com 
maintenance crews, maintenance plants and tools, energy supplies (normally, electricity or diesel), energy supply equipment, cargos, cargo loading equipment, passengers, passenger services (e.g. ticketing, inspection, safety and security) and passenger supplies (e.g. food, drinks, waste disposal equipment). Overall behaviour of a railway operation is unpredictable in detail. All railways experience, to a greater or lesser degree, unexpected delays and disruptions of service and occasional disasters due to unpredictable disruptive events such as changes in availability of resources due to resource failures, weather conditions or human errors. In addition transportation demands tend to change over time whilst changing transportation resources such as trucks is not always possible or practical.

The key task of railway management is the allocation of available transportation resources to transportation demands, aiming at achieving a complete match. The difficulty of this task primarily depends on the variability of demand and availability of resources, in particular, tracks, trains and human resources. As a rule, the variability is such that scheduling of resources must be done in real time.

\section{THE RAILWAY SCHEDULING PROBLEM}

The railway-scheduling problem can be formulated as the allocation of trains to track blocks with the aim of ensuring movement of trains according to specified constraints and priorities, where a block is a section of a railway line, which can be occupied only by one train. Standard operational conditions include the following:

- Different trains travel with different speeds.

- Different trains have different priorities.

- A train can overtake another train at a station or, if necessary, use a free track block on the parallel line (even on a line normally reserved for trains travelling in the opposite direction).

- The capacity of a station to hold trains, which are being overtaken, is limited.

Train scheduling is subject to the occurrence of unpredictable disruptive events, including train failures, track failures, train delays and track repair delays.

Whenever a disruptive event occurs, the schedule must be changed to accommodate the disruption with the minimal consequences to trains that are not directly affected.

\section{CASE STUDY 1}

Our task was to improve scheduling of trains on Moscow-St Petersburg main line, which is one of the busiest railway lines in Russia. The line has $700 \mathrm{~km}$ of tracks, 49 stations, 3,700 track blocks and 48 sections, including 2 four-track sections, 14 three-track sections, 31 double-track sections and 1 single-track section. The diversity of 810 trains is very high (passenger slow, passenger suburban, passenger express, passenger direct super express, freight, service, etc.) with 50 different priorities. An exceptional traffic density in suburban areas of Moscow and St Petersburg was a particular problem. Unpredictability of disruptions was very high: on average 100 unpredictable disruptive events are occurring during a single dispatcher shift.

A specific requirement was that the direct high-speed trains were not allowed to be late whatever the conditions. Track failures were frequent and repair works occasionally required up to $8 \mathrm{~h}$ of track closures. The official train timetable produced by railway headquarters was given to us as a reference and the starting point for real-time scheduling. 
Table 1: Case study 2: train delay reasons

\begin{tabular}{lcc}
\hline $\begin{array}{l}\text { Delay } \\
\text { Reasons }\end{array}$ & $\begin{array}{c}\text { Passenger train } \\
\text { delays p.a. }\end{array}$ & $\begin{array}{c}\text { Freight train } \\
\text { Delays p.a. }\end{array}$ \\
\hline Locomotive \& crew & 432 & 15819 \\
Passenger/freight carriages & 155 & 6659 \\
Track failures/bad conditions & 174 & 10667 \\
Signalling failures & 174 & 672 \\
Power supply failures & 99 & 451 \\
Wrong operational decisions & 442 & 667 \\
\hline
\end{tabular}

\section{CASE STUDY 2}

The second task was to develop a system of real-time schedulers for a Regional Siberian Railways with $5,000 \mathrm{~km}$ of tracks, carrying 5 millions of passengers and transporting 70 million tonnes of freight per annum (Table 1).

Trains often operate in this region under extreme weather conditions. The second case study is significant because of the volume of railway traffic in Russia. Russia is second only to China in railway traffic density in the world and third in railway traffic volume after the USA and China.

\section{THE SOLUTION}

\subsection{Brief overview}

The proposed solution for both case studies was a network of cooperating real-time schedulers. However, each network of schedulers required a considerable customization effort to accommodate specific client requirements.

The constituent schedulers of both networks are capable of entering the official train timetable as the initial state of the system, producing the schedule covering all constituent resources and then, most importantly, rapidly reacting to any unpredictable disruptive event, such as a failure or a delay, and rescheduling affected trains in real time ensuring a continuous strict correspondence between the schedule and the corresponding situation in the field.

The real-time scheduling of trains capable of rapidly eliminating consequences of unpredictable disruptions is our key achievement and represents, to the best of our knowledge, an original contribution to railway operation.

Our real-time schedulers enable to split railway timetabling into two parts:

- Producing master timetable applying evolutionary, heuristic or optimization methods, which can be used as a guide by railway authorities and users [11-13].

- Rapidly rescheduling master timetable whenever an unpredictable disruption occurs with the aim of eliminating consequences of disruptive events, as described in this paper. 


\subsection{Network architecture}

The architecture of the event-driven, multi-agent, real-time scheduling network is illustrated in Fig. 1.

The network consists of a number of real-time schedulers, which share the scheduling load and cooperate or compete with each other.

\subsection{Scheduler architecture}

Each scheduler consists of three key components: a knowledge base, a virtual world of software agents and the links between the virtual world and the real world.

Knowledge base consists of ontology, containing conceptual knowledge and databases containing factual knowledge.

Ontology contained classes of objects such as train, station, sidetrack and block, linked by relations, such as 'Train occupies Block', 'Train travels to Station', 'Sidetrack belongs to Station', etc. Each object was described by appropriate attributes.

Databases contain values defining instances of object classes.

Virtual world is the world of software agents. Typical agent types for the railway operation are train agent, station agent, sidetrack agent and block agent.

Scheduling is done by negotiation between demand agents and resource agents, where a typical demand agent is a train agent and a typical resource agent is a block agent. The basis for the negotiation is the premise that for a train to travel from A to B all blocks between these two points must be free from other trains at the time when the train is scheduled to reach them. When a train seeks a sidetrack at a station to wait to be overtaken by a higher priority train, resource agents involved in negotiation will be station agents and sidetrack agents. At any time there are over 40,000 agents simultaneously active.

Decisions on how to reschedule trains to eliminate consequences of disruptions are made by agent negotiations and conveyed to appropriate resources, as illustrated in Fig. 2.

The schedule is constructed in several stages.

The first stage is the production of a rough schedule for all trains based on the official timetable. The schedule is built for all trains in parallel, exposing points of conflicts between trains (more than one train on a block), if any.

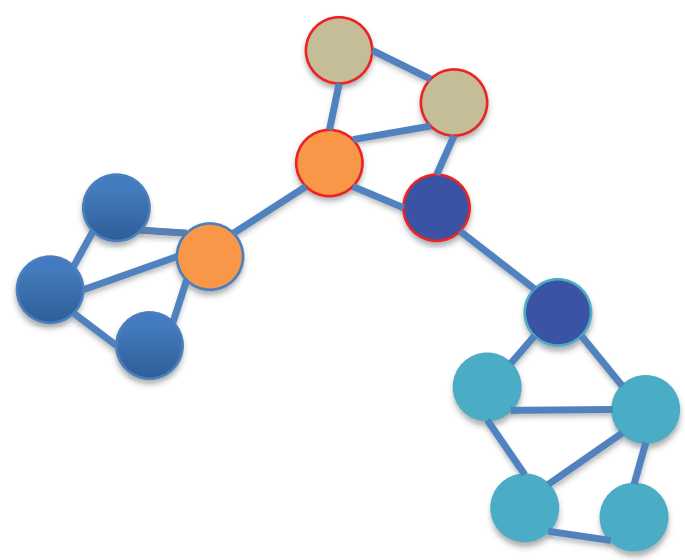

Figure 1: Scheduling of trains by a network of cooperating/competing schedulers. 


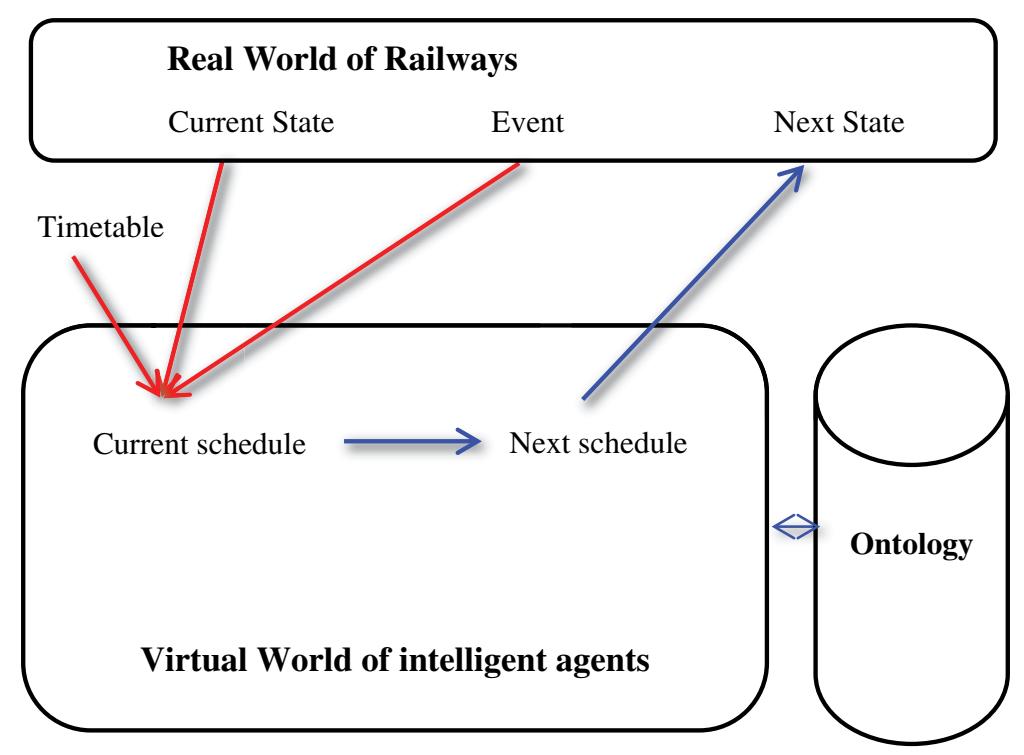

Figure 2: Real-time scheduler architecture.

The second stage is concerned with the elimination of conflicts. Train agents and block agents negotiate which of the trains that are in conflict will enter the contested block first, respecting, whenever possible, specified train priorities. Whenever a train has to overtake another train, the affected station agents and sidetrack agents get involved in the negotiation.

The third stage irons out anomalies, such as excessive delays imposed on some of the lowpriority trains, aiming to produce a well-balanced schedule.

All negotiations are conducted with the aim of maximizing enterprise value, which is a complex function that balances different, often conflicting, goals, i.e. respecting specified train priorities, minimizing train delays, minimizing the use of blocks of the line reserved for trains travelling in opposite direction, special train constrains, etc. In the third stage attempts to increase enterprise value are carried out until the process reaches the point of diminishing returns.

If the selected scheduling strategy does not lead to the satisfactory enterprise value, agents may autonomously decide to destroy the inadequate schedule and start the process from scratch, following alternative strategies (constructive destruction). The same may happen if the user of the system (a dispatcher) decides that the schedule produced by the agents is inadequate.

The scheduler receives inputs from and sends outputs to the railways information processing system.

\subsection{Salient features}

The constituent schedulers have the following salient features:

- Loading of the master timetable

- Loading of the maintenance plans

- Loading data from sensors on railway infrastructure and train positions

- Real-time processing of train locations and track occupancy

- Taking into account more than 80 criteria when scheduling trains (train length, weight, running/accelerating/braking times, track layouts, security, etc.) 
- Reacting in real time to unpredictable disruptive events (track or train failures, delays) and rescheduling affected trains

- Rapidly resolving unforeseeable conflicts (two trains demanding access to the same siding at the overlapping times) and rescheduling affected trains

- Visualization of output data to help dispatchers to make decisions

- Creation of and storing a variety of reports

\subsection{The key advantage of multi-agent technology}

Multi-agent schedulers are capable of rapidly (a) detecting a disruptive event, (b) searching for trains that will be affected and (c) rescheduling affected trains in real time before the next disruption occurs.

This unique feature is possible because computational search is replaced with search by interaction (communication) between agents.

\section{RESULTS}

The early version of the adaptive multi-agent scheduling system has substantially reduced the time required to reschedule the affected trains after the occurrence of disruptive events (failures or delays) and improved the utilization of railway resources. The following are some of the improvements achieved within operational and security constraints.

- Delays of high-speed trains practically eliminated

- Average train delays reduced to below $8 \%$

- Trains regained time lost due to disruptive events one and a half times faster

- Productivity of dispatchers doubled

- Losses due to delays reduced by $50 \%$

- Speed of rescheduling to eliminate consequences of failures increased dramatically (800 trains rescheduled within a minute)

- Customer satisfaction increased

- Opportunities opened for the increase of traffic density

\section{CONCLUSIONS}

Railway timetabling is a well-established art. However, recent developments in complexity science offer new superior methods for scheduling of trains in real time, which can increase profitability of railways and improve quality of service to railway clients. This paper describes how these new scheduling methods have been applied to two very different railway operations with a considerable success. To the best of authors' knowledge the real-time schedulers developed by the authors' teams are the first in the world actually working and delivering value to a railway authority under very difficult operating conditions.

The further work will include polishing agent negotiation and growing the network of competing/cooperating schedulers.

\section{REFERENCES}

[1] Prigogine, I., Is Future Given?, Singapore: World Scientific Publishing Co., 2003.

[2] Prigogine, I., The End of Certainty: Time, Chaos and the New Laws of Nature, New York: Free Press, 1997.

[3] Kaufman, S., At Home in the Universe: The Search for the Laws of Self-Organization and Complexity, Oxford, NY: Oxford Press, 1995. 
[4] Holland, J., Emergence: from Chaos to Order, Oxford, NY: Oxford University Press, 1998.

[5] Rzevski, G. \& Skobelev, P., Managing Complexity, WIT Press: Southampton, Boston, 2014.

[6] Rzevski, G., Soloviev, V., Skobelev, P., \& Lakhin, O., Complex adaptive logistics for the international space station. International Journal of Design \& Nature and Ecodynamics, 11(3), pp. 459-472, 2016. DOI: 10.2495/DNE-V11-N3-459-472.

[7] Rzevski, G., Knezevic, J., Skobelev, P., Borgest, N. \& Lakhin, O., Managing aircraft lifecycle complexity. International Journal of Design \& Nature and Ecodynamics, 11(2), pp. 77-87, 2016. DOI: 10.2495/DNE-V11-N2-77-87.

[8] Madsen, B., Rzevski, G., Skobelev, P., \& Tsarev, A., A strategy for managing complexity of the global market and prototype real-time scheduler for LEGO supply chain, International Journal of Software Innovation, 1(2), pp. 28-39, 2013. DOI: 10.4018/ ijsi.2013040103.

[9] Glaschenko, A., Ivaschenko, A., Rzevski, G. \& Skobelev, P., Multi-agent real time scheduling system for taxi companies. Proceedings of 8th International Conference on Autonomous Agents and Multiagent Systems (AAMAS 2009), eds. Decker, Sichman, Sierra, and Castelfranchi, Budapest, Hungary, May, 10-15, 2009,

[10] Andreev, S., Rzevski, G., Shveykin, P., Skobelev, P. \& Yankov, I., Multi-agent scheduler for rent-a-car companies. Lecture Notes in Computer Science, Volume 5696, Holonic and Multi-Agent Systems for Manufacturing: Forth International Conference on Industrial Applications of Holonic and Multi-Agent Systems, HoloMAS 2009, Springer: Linz, Austria, pp. 305-314.

[11] Tormos, P., Lova, A., Barber, F., Ingolotti, L., Abril, M. \& Salido, M. A., A genetic algorithm for railway scheduling problems. Studies in Computational Intelligence (SCI), 128, pp. 255-276, 2008. DOI: 10.1007/978-3-540-78985-7_10.

[12] Cai, X. \& Goh, C. J., A fast heuristics for the train scheduling problem. Computers and Operations Research, 50, pp. 738-753, 2002.

[13] Caprara, A., Monaci, M., Toth, P. \& Gvida, P., A Lagrangian heuristic algorithm for real-world train timetabling problem. Discrete Applied Mathematics, 154, pp. 738-753, 2006. DOI: 10.1016/j.dam.2005.05.026. 